\title{
Germanica
}

\section{L'analyse des discours officiels chez Christa Wolf, de Nachdenken über Christa T. à Kassandra}

Die «neue Sprache » bei Christa Wolf: Utopie und Wirklichkeit

\section{Michel Kauffmann}

\section{OpenEdition}

\section{Journals}

Édition électronique

URL : http://journals.openedition.org/germanica/2340

DOI : $10.4000 /$ germanica. 2340

ISSN : 2107-0784

Éditeur

Université de Lille

\section{Édition imprimée}

Date de publication : 30 décembre 1999

Pagination : 115-122

ISBN : 9782913857018

ISSN : 0984-2632

Référence électronique

Michel Kauffmann, «L'analyse des discours officiels chez Christa Wolf, de Nachdenken über Christa T. à Kassandra », Germanica [En ligne], 25 | 1999, mis en ligne le 30 janvier 2014, consulté le 06 octobre 2020. URL : http://journals.openedition.org/germanica/2340 ; DOI : https://doi.org/10.4000/ germanica. 2340

Ce document a été généré automatiquement le 6 octobre 2020.

(c) Tous droits réservés 


\section{L'analyse des discours officiels chez Christa Wolf, de Nachdenken über Christa T. à Kassandra}

Die «neue Sprache » bei Christa Wolf: Utopie und Wirklichkeit

Michel Kauffmann

1 Affirmant dans son intervention du 4 novembre 1989 « que toute révolution libère aussi le langage », Christa Wolf a replacé l'élément discursif au centre de la vie publique. Mais on sait que bien avant la Wende, son activité d'écrivain avait pris une dimension politique, la mettant souvent en conflit avec les autorités. On sera donc amené à rechercher dans son œuvre d'avant 1989 les premiers indices de cette interrogation sur le langage, vecteur et enjeu du politique.

\section{Nachdenken über Christa T. (1968-1969)}

2 La critique du discours dominant n'est pas explicitement le thème central de ce roman qui réactive une figure littéraire assez classique, celle de l'être sensible inapte à la vie en société. Lointaine émule de Werther ou de Madame Bovary, l'héroïne incarne les aspirations de la subjectivité face à un univers de déterminismes objectifs - ou se donnant pour tels. Forcément inadaptée, elle échouera dans sa tentative pour «dire je » par le biais de la création littéraire. Ce n'est cependant pas sans raison que ce roman a été reçu comme une critique du «Socialisme en construction» des années 50-601. Christa Wolf prend soin, en effet, de laisser quelques indices qui montrent bien que le conformisme auquel se heurte son héroïne est daté, historiquement spécifié, et que ce sont les formes et les contenus du discours officiel auxquels Christa T. ne parvient pas à s'adapter : « Sie hat gefühlt, wie die Worte sich zu verwandeln beginnen, wenn nicht mehr guter Glaube und Ungeschick und Übereifer sie hervorschleudern, sondern Berechnung, Schläue, Anpassungstrieb. » (NCT 71)². 
3 Dès Nachdenken se met donc en place ce qui constituera le ressort de la production ultérieure de Christa Wolf, le couple Sprachzweifel - Selbsterforschung ${ }^{3}$, interrogation sur le langage, contestation du discours ambiant, et recherche de soi dans et contre le langage. La critique du discours officiel, si l'on excepte la parenthèse de Kein Ort. Nirgends (1979) s'affirmera de plus en plus nettement, à mesure que l'écrivain s'efforcera d'accroître l'espace de liberté que lui concède le régime.

\section{Kindheitsmuster (1976)}

4 La réflexion sur le langage, esquissée dans Nachdenken über Christa T., s'amplifie dans Kindheitsmuster jusqu'à constituer une dimension essentielle de l'œuvre. Constamment, l'interrogation de la narratrice sur son passé fait ressurgir les mots qui ont balisé son parcours et dont elle déploie les connotations souvent troubles ou accusatrices. C'est ainsi que 8 chapitres sur 18, dans ce roman, débutent par l'analyse d'un fait de langue ou de discours, ou du moins par sa thématisation, suivant un procédé stylistique qui dans l'espace culturel germanophone est constitutif du genre de la Sprachglosse. Citons notamment :

Chap. 11 : « Endlösung.

Es ist nicht mehr festzustellen, wann du dieses Wort zum erstenmal gehört hast. » $(\text { KM } 315)^{4}$

Chap. 13 : «Dreizehn ist eine Unglückszahl.

Flucht wider Willen - auch eines der Stichworte, auf die ein Leben sich festlegen

ließe.» (KM 365)

Chap. $14:$ : Verfallen - ein deutsches Wort.

Blick in fremde Wörterbücher: Nirgends sonst diese vier, fünf verschiedenen Bedeutungen. Die deutsche Jugend ist ihrem Führer verfallen... » (KM 388)

À plusieurs reprises, l'auteur développe la thèse du pouvoir prégnant des mots ${ }^{5}$, et la biographie (partiellement autobiographique) de son héroïne Nelly empruntera les voies d'une archéologie des discours qui ont formé - à tous les sens du terme - cette jeune Allemande. Idiomatismes, lieux communs de la "sagesse populaire», mots tabous, propagande nazie, et plus tard, plus discrètement, langue de bois du communisme se relaient ou s'associent, tout au long du parcours de Nelly, pour l'entourer d'un « discours ambiant » oppressif et apparemment incontestable. La richesse du matériau linguistique collecté par Christa Wolf justifie une lecture documentaire de Kindheitsmuster, qui se définit comme une grande œuvre réaliste, si l'on veut bien inclure dans l'approche réaliste la perception des discours qui structurent une société. On pourra relever ainsi, sans prétention à l'exhaustivité, quelques exemples de ces discours.

Idiomatismes :

«Wenn über eine alte Geschichte endlich Gras gewachsen ist... » (KM 48)

« Es war kein Zuckerlecken... » (KM 57)

« Mein lieber Freund und Kupferstecher, das waren dir Heinis! » (KM 63)

« ... da kannst du Gift drauf nehmen » (KM 104)

" ... obwohl sie ihr gestohlen bleiben konnte » (KM 123)

«... und wenn es Schusterjungen regnet» (KM 153)

«Sie sollte um Himmels Willen die Kirche im Dorf lassen » (KM 223)

«... wegen der bekannten polnischen Wirtschaft » (KM 90)

« Wir sind doch hier nicht in der Judenschule » (KM 136) 
Ces expressions ont d'abord la fonction d'ancrer le récit et ses personnages dans une époque et un milieu social, celui de la petite bourgeoisie de l'entre-deux-guerres. À l'exception des deux derniers exemples cités, lourds d'antisémitisme et de nationalisme diffus, ce sont des faits de langue, ne présentant pas a priori de connotation idéologique, sinon précisément celle d'un usage non critique de la langue, conforme d'ailleurs aux compétences des locuteurs. Mais ce parler "spontané ", où les idiomatismes sont répétés plutôt que réappropriés, constituera aussi un terrain favorable pour un autre phénomène langagier, à mi-chemin entre les faits de langue et les faits de discours, et non exempt, lui, de charge idéologique : les proverbes et lieux communs de la « sagesse populaire ».

\title{
Les lieux communs :
}

\author{
«Aber Anlage bleibt Anlage » (KM 88) \\ « Pünktlichkeit ist das halbe Leben. Stillstand ist Rückschritt. Man muß aus seinem \\ Leben etwas machen » (KM 153) \\ «Morgen, morgen, nur nicht heute, sagen alle faulen Leute. Was du heute kannst \\ besorgen, das verschiebe nicht auf morgen. Langes Fädchen, faules Mädchen. Was \\ Hänschen nicht lernt, lernt Hans nimmermehr » (KM 270)
}

Ressortissant au genre des maximes et proverbes, ces énoncés - sur le conformisme profond desquels il n'est pas nécessaire d'insister - se donnent comme «ON-vrais », leur validité se fonde sur " l'effacement du sujet asserteur $»^{6}$ qui permet leur prise en charge par un « Volksempfinden » (KM 183) anonyme et omniprésent.

\section{Les maximes de la LTI}

9 C'est précisément cette forme de la maxime « ON-vraie » qu'emprunteront souvent les slogans nazis, par une habile adéquation du genre discursif aux attentes des allocutaires (ou faut-il dire co-énonciateurs?). Ils viendront ainsi s'amalgamer naturellement à ce fonds de conformisme, d'implicite partagé, qui constitue le lien social. Repris inconsciemment, ils finiront par ne plus se distinguer des anciens proverbes :

«Gemeinnutz geht vor Eigennutz » (KM 65)

«Seele ist Rasse von innen gesehen. Rasse ist Seele von außen gesehen » (KM 137)

«Ein deutsches Mädel weint nicht » (KM 179)

«Alle Juden sind Spekulanten » (KM 184)

« Wer nicht kämpft, verfault » (KM 203)

Cette archéologie des discours ambiants révèle ainsi que les manipulations de la propagande nazie exploitent les modalités discursives préexistantes (auxquelles on pourrait ajouter aussi celles de la publicité commerciale) plus qu'elles ne les modifient.

\section{La déconstruction des stéréotypes}

11 Mais toute archéologie débouche sur une déconstruction. Celle qu'entreprend Christa Wolf dans Kindheitsmuster s'appuie sur «l'ambiguïté des mots» («Doppelsinn der Wörter » KM 503 - un thème repris plusieurs fois dans le roman) pour procéder à une remotivation des métaphores, qui coïncident généralement avec les lieux communs du 
conformisme. Cette lecture qui fait éclater le sens convenu des stéréotypes est imputée aussi bien à la naïveté de Nelly enfant qu'à la sagacité de la narratrice adulte, instruite par les leçons de l'histoire :

«Alle diese nicht alltäglichen Vorkomnisse hatte man sich inmitten des Polnischen Korridors vorzustellen, der wegen der bekannten polnischen Wirtschaft niemals aufgeräumt sein konnte wie ihr eigener deutscher Korridor... » (KM 90) : Nelly enfant confond le couloir au sens géopolitique avec celui de la maison familiale, la tonalité humoristique du passage tourne en dérision, par contagion, le stéréotype raciste " polnische Wirtschaft».

13 " "Blinder Haß", ja das ginge, das wäre das einzig Richtige. Sehender Haß ist einfach zu schwierig. Der Führer muß sich blind auf euch verlassen können, darauf kommt es an. » (KM 185) : les sèmes négatifs de «blind» sont maladroitement réactivés par Nelly, à l'opposé de l'usage euphémisé du mot dans le discours nazi.

« Was ich nicht weiß, macht mich nicht heiß.

15 Was sie nicht wußten, machte sie lau » (KM 204) : par une syllepse de métaphore, « lau " (tiède) se déduit logiquement, au niveau du signifiant, de l'énoncé proverbial précédent, mais doit aussi se lire métaphoriquement, comme une caractérisation morale des parents de Nelly.

16 «Es ist nicht zu entscheiden, was zuerst dasein muß : die Bereitschaft vieler, aus ihrem Herzen eine Mördergrube machen zu lassen, oder Mordkisten, die durch die Landschaft fahren und aus Herzen Mördergruben machen » (KM 268) : le contexte est celui de l'« euthanasie » des malades mentaux, l'expression "Mördergrube », tirée de l'Evangile, au sens habituellement très atténué, est ici prise littéralement avec tout son poids d'accusation.

\section{La critique du discours stalinien}

17 Pour pertinente qu'elle soit, la déconstruction du discours nazi diffus n'avait rien qui pût opposer Christa Wolf à l'idéologie officielle de la RDA. Mais on peut déceler également dans Kindheitsmuster une critique, certes souvent discrète et indirecte, du stalinisme. Tout d'abord, l'aisance avec laquelle son héroïne, jeune hitlérienne convaincue jusqu'en mai 1945, se met au service des occupants américains, puis soviétiques, a quelque chose de gênant. D'autres notations évoquent la nécessité, pour les Allemands de sa génération, d'affronter aussi leur passé stalinien. La base logique, implicite ou même explicite, de l'argumentation sera celle d'un rapprochement, d'une continuité possibles entre les deux périodes: «Den Blicken der Kinder nicht ausweichen müssen, ... wenn von... "früher" die Rede ist : Früher in den dreißiger, früher, in den fünfziger Jahren.» (KM 202). Le lecteur reconnaîtra ces décennies comme emblématiques des dictatures nazie ou stalinienne, des «deux couches de chants " (KM 517) qui ont marqué une génération. Un rêve à tonalité funèbre, où apparaît une stèle au nom de Staline (KM 332), dont la mort est reçue avec incrédulité par les personnages du rêve, amène la narratrice à s'interroger : «Wann... werden wir auch darüber zu reden beginnen? Das Gefühl loswerden, bis dahin sei alles, was wir sagen, vorläufig und dann erst werde wirklich gesprochen werden? ». L'anaphorique darüber, certes assez flou (peut-être par souci de la censure) ne peut renvoyer qu'à la problématique précédemment évoquée, celle du stalinisme. Cette remise en question 
du passé stalinien est donnée par la narratrice, porte-parole de l'auteur, comme la condition d'une "vraie parole ": c'est peut-être la première thématisation, chez Christa Wolf, de l'exigence d'une "libération du langage", qui aboutira à son intervention du 4 novembre 1989. Un passage enfin persifle discrètement la « langue de bois » règnant toujours en RDA à l'époque de la rédaction du roman. Une cérémonie officielle est décrite en phrases pour la plupart dénuées de verbe, d'adjectifs ou de modalisateurs, et dont la neutralité voulue traduit l'absence d'engagement personnel de la part de la narratrice. La conclusion du passage - "Redefetzen vom nahen Versammlungsplatz her, russisch und deutsch. Vorgefertigte Satzteile aneinandergefügt » (KM 531) - pointe un trait caractéristique, souvent relevé, de la langue de bois : la tendance à la stéréotypie et à la locutionnalisation.

\section{Kassandra (1983)}

En reprenant, comme Brecht ou Heiner Müller avant elle, un thème tiré de l'antiquité, Christa Wolf pouvait exploiter les ressources de la polyisotopie ${ }^{7}$ : l'attente du lecteur le prédispose à un lecture à plusieurs niveaux, où les épisodes tirés d'Homère sont interprétés comme référant indirectement à la réalité de la RDA des années 80 . De fait, l'enchaînement funeste qui conduit à la destruction de Troie est imputé au personnage, créé par Christa Wolf, d'Eumelos, qui prendra le pouvoir réel à la faveur de la guerre et dont les méthodes sont celles de la dictature moderne. C'est ainsi que son "Sicherheitsnetz $»^{8}$ (K 118) - allusion évidente à la Stasi - s'étend peu à peu à toute la ville. La population est étroitement contrôlée par ses agents. Surtout, l'opinion est manipulée par un discours officiel orienté et encadré par des "Sprachregelungen » (K 77) euphémistiques ou péjorantes. L'échec des missions diplomatiques troyennes (les «trois navires ») est ainsi travesti en succès, la population préparée à la guerre par un "Sprachkrieg» (K 76) savamment orchestré : «Ich war Zeugin, wie... eine Nachricht hergestellt wurde... Jubelnd lief das Volk durch die Straßen. Ich sah eine Nachricht zur Wahrheit werden. » (K 76). Le discours se substitue finalement à la réalité, le faux est indiscernable du vrai («Was öffentlich geworden ist, ist auch real» (K 98)), Troie devient une «ville fantôme " (« Geisterstadt » K 99). Le mensonge qui symbolise cette perversion du sens, c'est l'absence d'Hélène (dans l'interprétation de Christa Wolf, Paris n'est pas parvenu à amener celle-ci à Troie), que l'on cache à la population, condamnée de ce fait à «lutter pour un fantôme » (K 81). On voit que dans Kassandra, la question des manipulations du discours atteint une dimension quasi-métaphysique. De même, le contre-discours capable de s'opposer à ce langage du pouvoir - identifié par l'auteur au principe masculin même - se situera du côté de la parole des femmes, symbolisée par les mythes matriarcaux et le culte de Cybèle, mais inscrite aussi dans les «messages du corps » qui viennent hanter la prophétesse : «Wie jedem Menschen gab mir der Körper Zeichen; anders als andere war ich nicht imstande, die Zeichen zu übergehn » (K 68). Comme dans Nachdenken über Christa T., la résistance au discours du pouvoir prend ainsi la forme d'une revendication des droits de la subjectivité.

À la veille de la Wende, Christa Wolf est ainsi parvenue à une dénonciation, en termes très vigoureux, de l'oppression politique règnant en RDA. Le travestissement antique, dernière concession à la censure, est des plus transparents. Surtout, la mise en évidence de la fonction politique du discours apparaît comme une dimension fondamentale de sa réflexion et de son écriture. Si elle reste largement indirecte, dans 
Nachdenken über Christa T. par le biais de l'évocation des difficultés qu'éprouve son héroïne à articuler son malaise, elle procède de façon ample, étayée par une foule d'observations de détail, dans Kindheitsmuster, qui expose avec une rigueur quasiclinique les déformations que subit la conscience d'une jeune enfant immergée dans le bain linguistique et idéologique du $3^{\mathrm{e}}$ Reich. Le stalinisme, dans ce roman, est cependant évoqué en creux, par allusion, plutôt qu'affronté. Il faudra attendre Kassandra pour que soit thématisé le lien entre État policier et manipulation du langage.

\section{NOTES}

1. Mohr, H., «Die zeitgemäße Autorin - Christa Wolf in der DDR » in Mauser, W (Hrsg.) : Erinnerte Zukunft - 11 Studien zum Werk Christa Wolfs, Königshausen+Neumann, Würzburg, 1985.

2. Wolf, C., Nachdenken über Christa T., Luchterhand, Neuwied, 1970.

3. Jäger, M., « Die Grenzen des Sagbaren. Sprachzweifel im Werk von Christa Wolf» in Drescher A. : Christa Wolf, ein Arbeitsbuch, Luchterhand Literaturverlag, Frankfurt/Main, 1990.

4. Wolf, C., Kindheitsmuster, Sammlung Luchterhand, Luchterhand Literaturverlag, Hamburg, 1979.

5. «Und die Einsicht, daß die Sprache, indem sie Benennungen erzwingt, auch aussondert, filtert : im Sinne des Erwünschten. Im Sinne des Sagbaren. Im Sinne des Verfestigten. » (KM 312).

6. Maingueneau D., L'analyse du discours. Introduction aux lectures de l'archive, Hachette, Paris, 1991. (p. 118).

7. Maingueneau D., Pragmatique pour le discours littéraire, Bordas, Paris, 1990. (p. 47-48).

8. Wolf, C., Kassandra, Sammlung Luchterhand im dtv, München, 1993.

\section{RÉSUMÉS}

L'analyse critique du discours officiel apparaît comme une dimension importante, voire fondamentale, dans quatre textes narratifs de Christa Wolf: dans Nachdenken über Christa T. (1968), sous la forme d'un conflit entre la subjectivité individuelle et le conformisme ambiant, dans Kindheitsmuster (1976), par la description de la banalisation de la Lingua Tertii Imperii, relayée insensiblement dans les façons de parler quotidiennes, mais aussi par l'évocation du passé stalinien de la RDA, dans Kassandra (1983), où les techniques de la propagande moderne sont dénoncées sous un travestissement antique, et enfin dans Was bleibt (1990), décrivant la dégradation de la personne lorsqu'elle est soumise à un contrôle policier omniprésent. Malgré tout, on voit se profiler l'utopie d'un nouveau langage fondée sur le dialogue entre partenaires égaux, utopie qui se réalisera un court moment pendant la révolution pacifique de l'automne 1989, comme en témoigne le discours Sprache der Wende du 4 novembre 1989. 
Die kritische Analyse des herrschenden Diskurses erscheint als eine bedeutende, wenn nicht sogar konstitutive Dimension in vier Prosatexten von Christa Wolf : in Nachdenken über Christa T. (1968), in der Form eines Konfliktes zwischen Subjektivität und Konformismus, in Kindheitsmuster (1976) als Beschreibung der Verbreitung der Lingua Tertii Imperii durch deren unreflektierte Wiederaufnahme in der Alltagssprache, aber auch als Thematisierung der stalinistischen Vergangenheit der DDR, in Kassandra (1983), wo unter der antiken Maske die moderne Propagandatechnik beschrieben wird, und schließlich in Was bleibt (1990), als Darstellung der Pervertierung des Individuums durch ein allgegenwärtiges Überwachungssystem. Jedoch erscheint am Horizont die Utopie einer auf dem Gespräch zwischen gleichberechtigten Partnern gegründeten neuen Sprache. Diese Utopie scheint eine kurze Zeit lang während der friedlichen Revolution vom Herbst 1989 Wirklichkeit geworden zu sein, wie Christa Wolfs Rede Sprache der Wende vom 4. November 1989 es bezeugt.

\section{AUTEUR}

\section{MICHEL KAUFFMANN}

Université de Paris III 